\title{
SPILOTYLENCHUS ARTHURI, gen. n., sp. n. (NEMATODEA, TYLENCHIDA : Allantonematidae), PARASITE DE SPILOPSYLLUS CUNICULI (Dale, 1878) (Siphonaptera : Pulicidae) $^{1}$
}

\author{
H. LAUNAY*, J. DEUNFF** et 0. BAIN $^{* * *}$
}

\begin{abstract}
RÉSUMÉ. Spilotylenchus arthuri gen. n., sp. n. (Nematodea, Tylenchida : Allantonematidae) parasite du Siphonaptère Spilopsyllus cuniculi (Dale, 1878) inféodé au lapin de garenne, est décrit à partir d'un matériel provenant de la région parisienne et comprenant des femelles parasites et des adultes, mâles et femelles, libres. Neoparasitylenchus megabothridis Laumond et Beaucournu, 1977 et $N$. laplandicus Rubtzov et Darskaya, 1981, sont considérés comme appartenant à ce nouveau genre. $S$. arthuri provoque un blocage de l'oogénèse chez les puces femelles.
\end{abstract}

\section{Spilotylenchus arthuri, gen. n., sp. n. (Nematodea, Tylenchida : Allantonematidae), parasite of Spilopsyllus cuniculi (Dale, 1878) (Siphonaptera : Pulicidae) $^{1}$}

SUMMARY. Spilotylenchus arthuri gen. n., sp. n. (Nematodea, Tylenchida: Allantonematidae) parasite of the European rabbit flea Spilopsyllus cuniculi (Dale, 1878) is described from a material including parasitic females so as freeliving adults, male and female. Neoparasitylenchus megabothridis Laumond et Beaucournu, 1977 and N. laplandicus Rubtzov et Darskaya, 1981, are held to belong to this new genus. S. arthuri produce a locking of the oogenesis in female fleas.

Les puces du lapin de garenne jouent un rôle actif dans l'épidémiologie de la myxomatose. A cet égard, dans le cadre d'une action pluridisciplinaire menée en faveur du lapin en France, l'étude de ces vecteurs a été entreprise et des mesures prophylactiques envisagées. Parmi ces mesures la lutte biologique est possible contre Spilopsyllus cuniculi qui est le Siphonaptère du lapin le plus répandu : en effet S. cuni-

I. Travail effectué grâce à l'aide financière de l'A.N.C.L.A.T.R.A. et à des subventions de l'Office National de la Chasse.

* Institut de Parasitologie de l'Ouest, Faculté de Médecine, Avenue du Professeur Léon Bernard, F 35043 Rennes Cedex.

** Laboratoire de Parasitologie (Entomologie médicale), même adresse.

*** Laboratoire des Vers, Muséum National d'Histoire Naturelle, Paris.

Accepté le r6 septembre 1982. 
culi est parasité par deux tylenchides Allantonematidae qui bloquent l'oogénèse chez les puces femelles. Nous décrivons ici l'un de ces deux Nématodes et nous sommes heureux de dédier ce taxon à Christian Philippe Arthur (ingénieur, Office National de la Chasse) en hommage à ses recherches sur le lapin de garenne et en témoignage d'amitié.

\section{I - Matériel et méthodes}

\section{A - Origine du matériel}

Les Spilopsyllus cuniculi ont été récoltés sur des lapins tués à la chasse à Dourdan (Yvelines). Lors des premières collectes les puces ont été fixées sur le terrain en alcool à $70^{\circ}$. Par la suite ce matériel a pu être conservé en tubes de survie et ramené vivant au laboratoire.

\section{B - Méthodes d'étude}

1 - Isolement et obtention des différents stades du Tylenchide

a) à partir des puces fixées en alcool

Spilopsyllus cuniculi étant une puce très sclérifiée le repérage des individus parasités est quasiment impossible mis à part quelques spécimens hyperparasités dont l'aspect dilaté est caractéristique. Il est donc nécessaire de disséquer la plus grande partie des puces récoltées, pour mettre en évidence la présence des Tylenchides (femelles gravides et larves à différents stades). La fixation à l'alcool provoque une adhérence des nématodes au contenu abdominal de la puce et les fragilise. Cependant nous avons pu tirer le meilleur parti de ce matériel en adaptant un traitement utilisé en acarologie pour les spécimens conservés depuis longtemps en alcool : le "Nesbitt's fluid " (in Krantz, 1978). Par la suite les nématodes sont alors traités de la même manière que le matériel vivant.

b) à partir de puces non fixées

Le repérage à frais, in vivo, permet de mettre en évidence chez les puces de nombreux parasites et en particulier des Tylenchides (Beaucournu et Deunff, 1975). Chez $S$. cuniculi comme nous l'avons vu précédemment, cette technique est difficilement applicable.

- Les puces hyperparasitées, plus facilement repérables sont toutefois séparées des autres puis disséquées dans du Ringer pour Insectes à 7\% (formule de Novak). Les Tylenchides femelles parasites et une partie des larves sont fixés et montés. L'autre partie est mise en élevage ; l'évolution vers les stades adultes libres est obtenue en quelques jours, à la température du laboratoire, en baissant la salinité, toutes les 24 heures, de $7 \%$ à $5 \%$ puis $3 \%$. La fixation et le montage de ces stades adultes s'échelonnent sur plusieurs semaines afin de pouvoir étudier les différents stades de maturation. 
- Les autres puces, chez lesquelles le parasitisme n'est pas évident, sont gardées en. tubes de survie et contrôlées journellement pour surveiller les sorties de Tylenchides libres qui sont alors mis en élevage directement en Ringer à $3 \%$. Les puces mortes sont enlevées au fur et à mesure et conservées dans du Ringer à $3 \%$ où l'on obtient encore la sortie de stades libres.

2 - Fixation, montage et observation

a) Observation à frais

Le tylenchide est observé in vivo entre lame et lamelle dans une goutte de Ringer à $3 \%$.

b) Observation après coloration

Le tylenchide est placé entre lame et lamelle dans une solution à $0,1 \%$ de New Blue R puis fixé en chauffant la lame sur une flamme jusqu'à ébullition. Le colorant pénètre alors instantanément dans le nématode et augmente les contrastes cellulaires.

c) Observation après fixation et montage ${ }^{2}$

Les spécimens vivants sont fixés à chaud $\left(80^{\circ} \mathrm{C}\right)$ dans la solution ci-dessous puis gardés au saturateur (même solution que le fixateur) pendant 48 heures.

Formol du commerce $(40 \%) \ldots \ldots \ldots \ldots \ldots \ldots \ldots . \ldots \ldots$

Acide acétique cristallisable .............. 1,5 cc

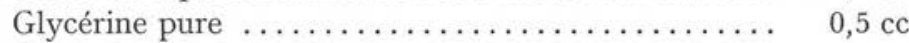

Acide picrique en solution saturée $\ldots \ldots \ldots \ldots \ldots \ldots$ gouttes

Eau distillée ..................... 43 cc

Après évaporation en étuve à $37^{\circ}$ le matériel subit une dessication par une solution d'alcool glycériné (Éthanol 960 : $20 \%$, Glycérine pure : $1 \%$, eau distillée : $79 \%$ ) dans un saturateur à alcool, en étuve à $37^{\circ}$. Cette dessication est répétée une seconde fois 24 heures plus tard.

Le matériel est monté entre lame et lamelle dans la glycérine.

Les spécimens colorés au New Blue $\mathrm{R}$ sont démontés et postfixés en suivant la même technique.

\section{II - Spilotylenchus arthuri gen. n., sp. n.}

\section{A - Spilotylenchus gen. $\mathbf{n}$.}

Allantonematidae (Pereira, 1931), Chitwood et Chitwood, 1937, nous caractérisons Spilotylenchus, gen. n., par une seule génération de femelles, possédant un stylet, les femelles parasites étant ovovivipares, arquées dorsalement, à maturité leur corps est occupé en grande partie par l'utérus renfermant une multitude de petites larves.

2. Techniques communiquées par C. Laumond, Station de Recherches sur les Nématodes, I.N.R.A., Antibes. 
Chez l'insecte on ne trouve que des femelles parasites et des larves; les femelles libres sont seules infestantes, l'habitus est rectiligne, l'ovaire composé de quelques cellules alignées, la vulve postérieure. Les mâles libres présentent un petit stylet, un habitus rectiligne post mortem, deux spicules arqués, le gubernaculum est présent, la bursa pélodère.

\section{- Discussion}

Tous les caractères de cette espèce nouvelle la rapprochent de Neoparasitylenchus megabothridis Laumond et Beaucournu, 1977 et peut-être de $N$. laplandicus Rubtzov et Darskaya 1981, mais cette dernière espèce étant décrite uniquement à partir de femelles parasites à un stade jeune, son statut sera peut-être à revoir. Les femelles parasites de ces trois espèces sont ovovivipares, ce qui les sépare du genre Contortylenchus, dont les femelles sont ovipares, et toutes trois à courbure dorsale ce qui les écarte du genre Neoparasitylenchus dont les femelles ont une courbure ventrale (clé de Poinar, 1975). Nous sommes donc amenés à les placer dans le genre que nous décrivons ici : Spilotylenchus megabothridis (Laumond et Beaucournu, 1977) comb. nov., Spilotylenchus laplandicus (Rubtzov et Darskaya, 1981) comb. nov.

\section{B - Espèce type : Spilotylenchus arthuri sp. n. \\ 1 - Matériel}

- Holotype : femelle parasite, allotype : mâle adulte libre.

— Paratypes : 16 femelles parasites, 13 mâles adultes libres, 13 femelles adultes libres.

Tous ces spécimens, obtenus à partir de Spilopsyllus cuniculi, en provenance de Dourdan (Yvelines, France).

Holotype, allotype et 39 paratypes sont déposés dans les collections du Laboratoire de Parasitologie (Entomologie médicale) de la Faculté de Médecine de Rennes. 3 paratypes ( 1 femelle parasite, 1 mâle adulte libre, 1 femelle adulte libre) sont déposés au Laboratoire des Vers du Muséum National d'Histoire Naturelle de Paris. Holotype et allotype seront ultérieurement déposés dans ce dernier laboratoire.

\section{2 - Description}

- Femelles parasites gravides

Longueur totale : moyenne $1127 \mu \mathrm{m}$ (905 à 1280), D.S. (Déviation Standard) = 95, Holotype : $1079 \mu \mathrm{m}$. Largeur maxima : moyenne $92 \mu \mathrm{m}$ (75 à 116). D.S. = 13, Holotype : $75 \mu \mathrm{m}$. Distance de l'apex au pore excréteur : moyenne $31 \mu \mathrm{m}$ (19 à 47), D.S. $=6$, Holotype : $32 \mu \mathrm{m}$. Longueur de la queue (depuis l'anus jusqu'à l'extrémité postérieure) : 8 à $16 \mu \mathrm{m}$ (Holotype : $16 \mu \mathrm{m}$ ) ; queue conique, souvent rétractée et plissée, mucron généralement bien marqué ( fig. 1 et 3). Distance de la vulve à l'extrémité postérieure : moyenne $37 \mu \mathrm{m}$ (23 à 46), D.S. $=5$, Holotype : $42 \mu \mathrm{m}$; vulve sans lèvres (fig. 3). Cuticule lisse, non plissée, y compris dans la zone circum-orale qui est relativement étroite. Ovaire, réfléchi plusieurs fois sur lui-même, et spermathèque, arrondie, très antérieurs et repoussés graduellement par l'utérus qui se remplit d'œufs 


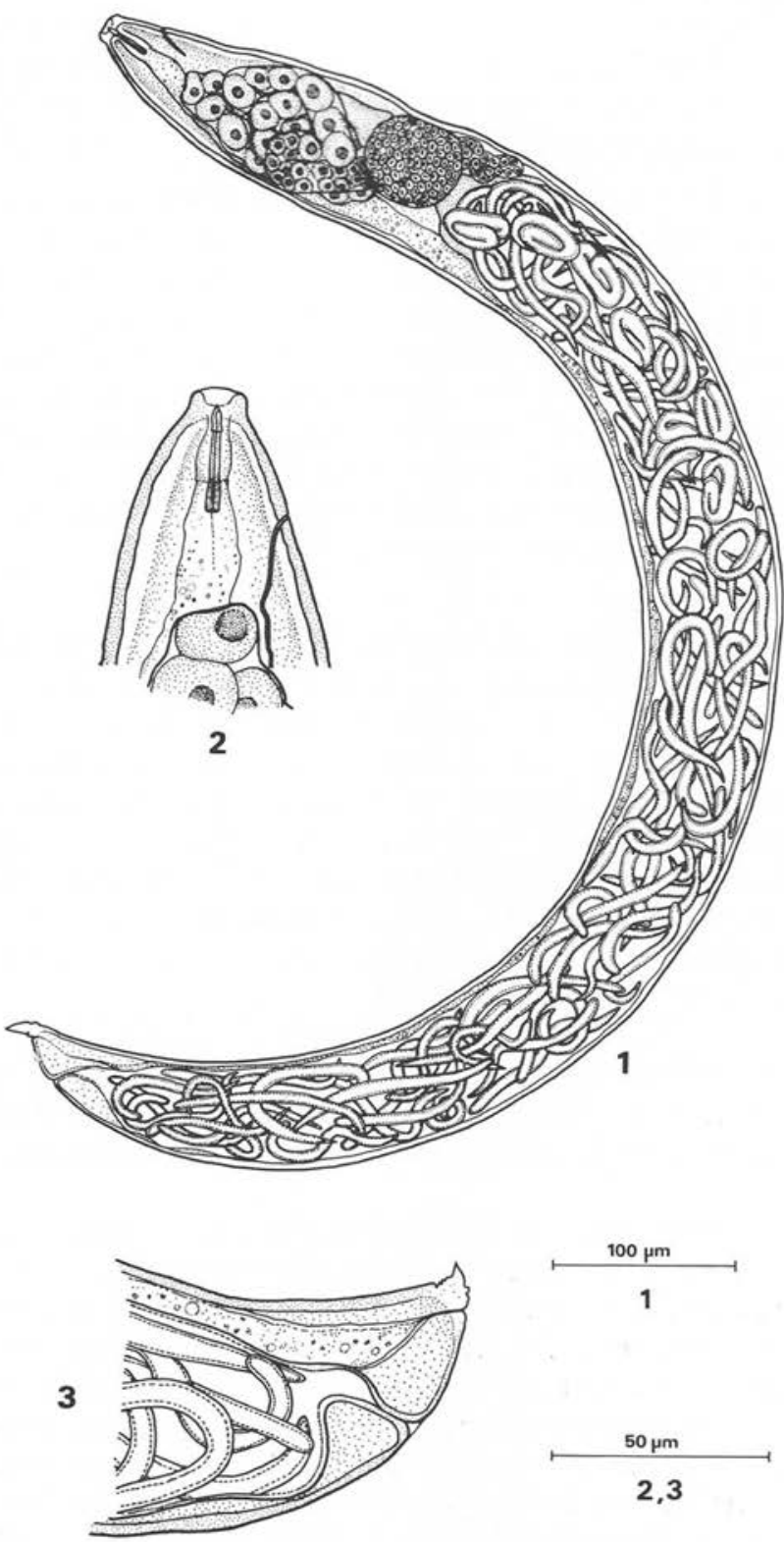

FIG. I-3. - Spilotylenchus arthuri gen. n., sp. n., femelle parasite. I : holotype, vue latérale droite; 2 : holotype, partie antérieure; 3 : paratype, partie postérieure. 
et de larves ; rapport longueur de l'utérus sur longueur totale : moyenne 0,72 (0,59$0,81)$, D.S. $=0,07$, Holotype : 0,71. Stylet 24 à $26 \mu \mathrm{m}$, caractéristique, en forme de flèche (fig. 2), la pointe apicale est conique avec une ouverture ventrale non terminale, la hampe est rectiligne, symétrique, élargie dans sa moitié postérieure.

\section{- Femelles adultes libres ${ }^{3}$}

Cuticule avec de fines striations transversales. Longueur totale : moyenne $661 \mu \mathrm{m}$ (546 à 749), D.S. = 79. Largeur maxima : moyenne $25 \mu \mathrm{m}(22-34)$, D.S. $=3$. Distance de l'apex au pore excréteur : moyenne $51 \mu \mathrm{m}$ (40-59), D.S. $=6$. Distance de l'apex à l'anneau nerveux : moyenne $71 \mu \mathrm{m}$ (61-82), D.S. $=6$. Longueur de la queue : moyenne $34 \mu \mathrm{m}$ (29-46), D.S. = 6. Distance de la vulve à l'extrémité postérieure : moyenne $65 \mu \mathrm{m}$ (52-89), D.S. = 11. Même stylet que celui de la femelle parasite (fig. 6). 3 à 6 oocytes à l'ovaire, oviducte étroit, générallement rectiligne, spermathèque plus ou moins différenciée selon le degré de maturité (fig. 5 et 7). Utérus rectiligne à cellules indistinctes, vagin perpendiculaire à la paroi du corps, vulve avec des lèvres marquées et à ouverture transversale ( fig. 4 et 5 ).

- Mâles adultes libres ${ }^{4}$

Cuticule finement striée. Longueur totale : moyenne $585 \mu \mathrm{m}(505-700)$, D.S. $=61$, allotype : $570 \mu \mathrm{m}$. Largeur maxima : moyenne $19 \mu \mathrm{m}(17-23)$, D.S. $=2$, allotype : $18 \mu \mathrm{m}$. Distance de l'apex au pore excréteur : moyenne $46 \mu \mathrm{m}$ (39-62), D.S. $=8$, allotype $50 \mu \mathrm{m}$. Distance de l'apex à l'anneau nerveux : moyenne $57 \mu \mathrm{m}$ (50-73), D.S. $=8$, allotype : $60 \mu \mathrm{m}$. Distance de l'extrémité des spicules jusqu'à la pointe postérieure : moyenne $34 \mu \mathrm{m}(27-44)$, D.S. $=5$, allotype : $37 \mu \mathrm{m}$. Spicules arqués (fig. 10), long de $16 \mu \mathrm{m}$ en moyenne (14 à 18) (allotype $16 \mu \mathrm{m})$. Gubernaculum réduit (3 à $4 \mu \mathrm{m}$ ). Bursa pélodère ( $f i g$. 11), longueur moyenne $53 \mu \mathrm{m}$ (44-71), D.S. $=8$, allotype : $51 \mu \mathrm{m}$. Petit stylet en forme de flèche (fig. 9) long de 7 à $8 \mu \mathrm{m}$.

\section{3 - Diagnose}

\section{a) Femelle parasite gravide}

laplandicus (longueur 1500 à $1550 \mu \mathrm{m}$, stylet 19 à $22 \mu \mathrm{m}$, d'après Rubtzov, 1981) se sépare de arthuri et de megabothridis par sa taille plus importante et son stylet plus petit.

arthuri et megabothridis ont des mensurations assez proches mais le stylet d'arthuri est en général plus grand et de forme différente dans sa partie postérieure : il est asymétrique chez megabothridis, symétrique chez arthuri. D'autre part, quand l'utérus atteint son maximum d'extension (c'est-à-dire quand il est rempli de larves), celui-ci occupe environ les trois quarts du corps chez arthuri, les deux tiers chez megabothridis. Rubtzov indique pour laplandicus que l'utérus occupe moins de la moitié du corps, il faut toutefois remarquer qu'il s'adresse à un stade de maturation précoce.

Si nous suivons Laumond et Beaucournu, 1978 repris par Rubtzov, 1981, megabothridis et arthuri seraient séparables sur la forme différente de l'extrémité posté-

3. Mensurations effectuées sur des individus obtenus en condition d'élevage.

4. $I d$. 

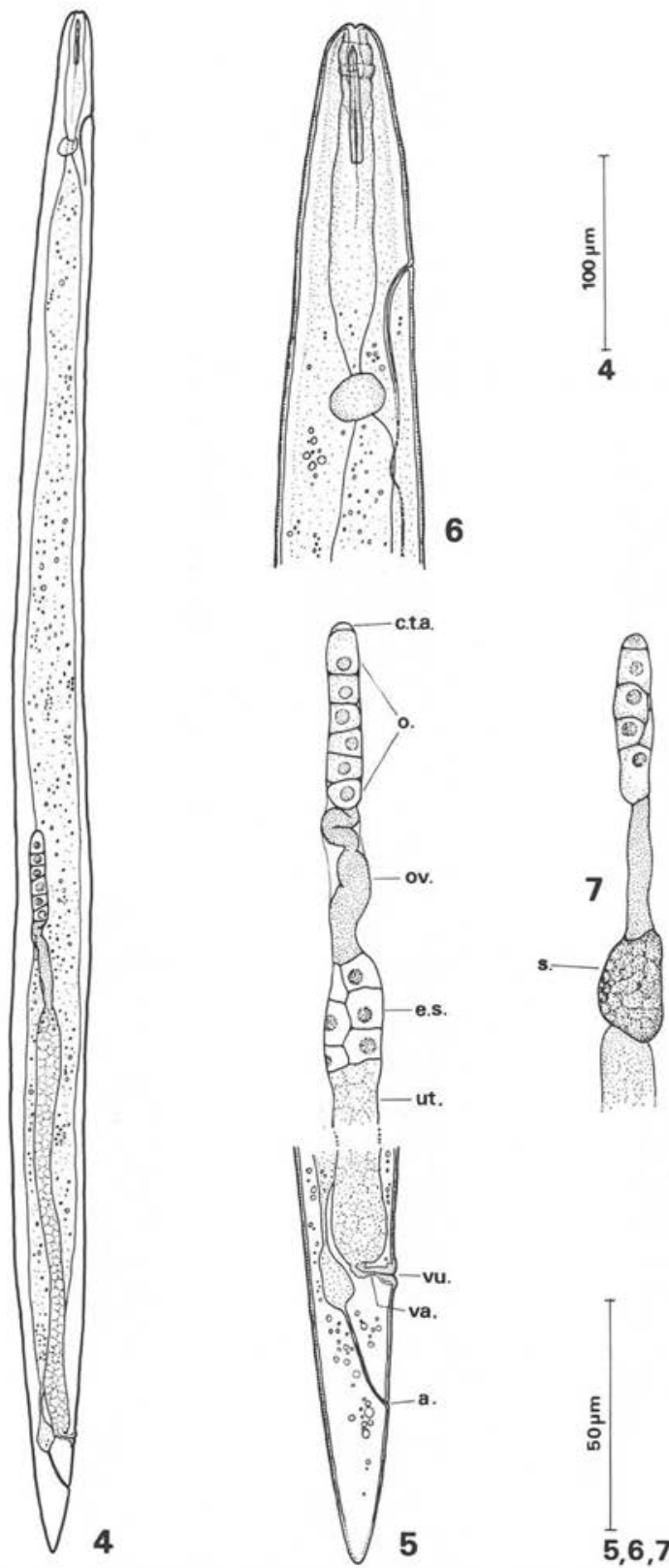

Fig. 4-7. - Spilotylenchus arthuri gen. n., sp. n., femelle adulte libre. 4 : paratype, vue latérale droite ; $5:$ paratype, partie postérieure et gonade ; $6:$ paratype, partie antérieure ; $7:$ paratype, gonade.

$\mathrm{a}=$ anus, c.t.a. $=$ cellule terminale apicale, e.s. $=$ ébauche de la spermathèque, $0 .=$ oocytes, ov. $=$ ovaire, ut. $=$ utérus, va. $=$ vagin, vu. $=$ vulve. 


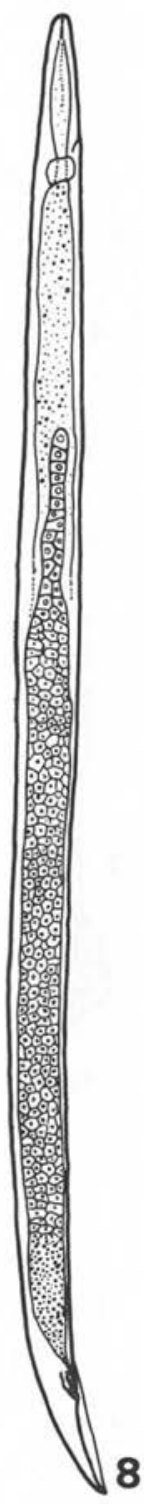

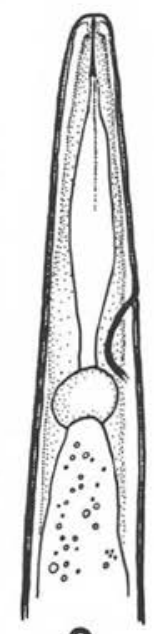

9
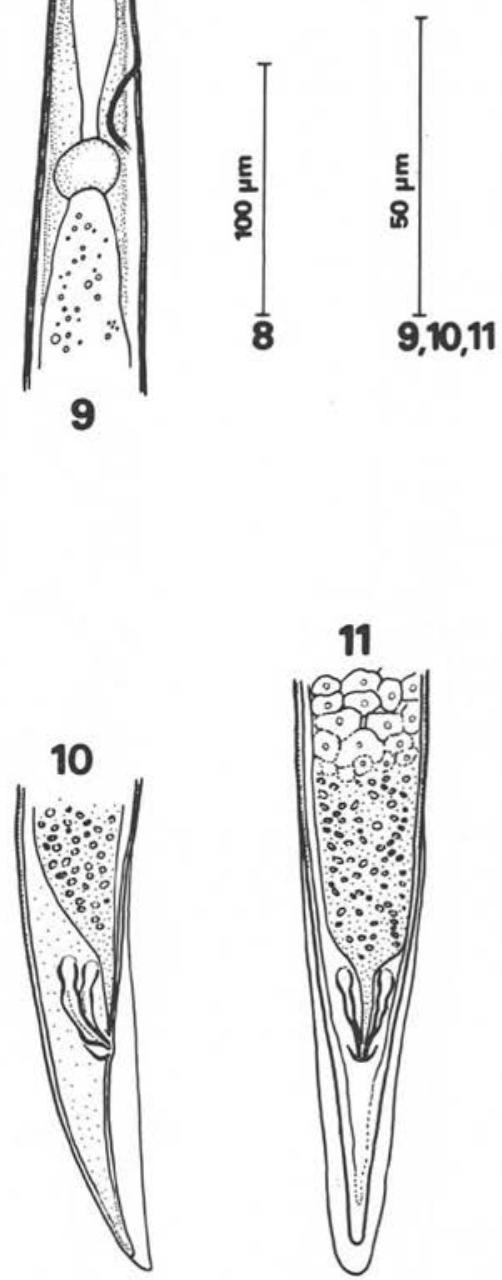

Fig. 8-Ir. - Spilotylenchus arthuri gen. n., sp. n., mâle adulte libre. 8 : paratype, vue latérale droite; 9 : paratype, partie antérieure; ro : paratype, partie postérieure, vue latérale; II : allotype, partie postérieure, vue ventrale. 
rieure : la queue est arrondie chez megabothridis, alors que chez arthuri elle est pointue avec un mucron bien marqué. Nous n'avons pas retrouvé ce caractère sur les megabothridis que nous possédons dans nos collections (provenance Megabothris turbidus, St-Renan, Finistère, France), en effet elles présentent une extrémité postérieure pointue ce qui semble logique puisque ces mêmes femelles, à l'état libre, avaient la queue pointue.

b) Stades adultes libres

Les stades libres de laplandicus n'étant pas décrits, nous ne considérons ici que arthuri et megabothridis (voir mensurations in Laumond et Beaucournu, 1978).

Les mâles sont immédiatement séparables par la longueur du stylet, du gubernaculum, la distance de l'extrémité antérieure à l'anneau nerveux. Les femelles se séparent également par la distance de l'extrémité antérieure à l'anneau nerveux, par la distance de la vulve à l'extrémité postérieure et par la taille du stylet généralement plus petit chez megabothridis.

Mâles et femelles d'arthuri sont en moyenne plus petits que ceux de megabothridis (longueur totale, largeur, longueur de la queue) et présentent généralement un dimorphisme dans la taille (les mâles sont plus petits que les femelles).

\section{C - Relations hôte-parasite}

\section{1 - Spécificité}

Nous considérons Spilotylenchus arthuri comme un parasite spécifique de Spilopsyllus cuniculi pour plusieurs raisons :

- les autres espèces de puces récoltées sur micromammifères dans les zones où $S$. arthuri a été trouvé, n'avaient jamais ce parasite.

- S. cuniculi est écologiquement complètement isolé et il n'y a pas d'interactions avec d'autres siphonaptères :

- S. cuniculi est la seule puce spécifique du lapin dans la région considérée ${ }^{5}$.

- Il n'y a pas de fréquentation ou d'occupation des nids de lapins (où se reproduit $S$. cuniculi et où se trouvent ses larves et ses nymphes) par d'autres mammifères, donc pas de contamination par d'autres puces que celles du lapin.

La spécificité présente un intérêt pratique car elle offre une bonne indication en ce qui concerne la diagnose des femelles parasites : megabothridis est considéré comme spécifique de Megabothris turbidus, laplandicus a été trouvé chez Megabothris rectangulatus, enfin arthuri est spécifique de Spilopsyllus cuniculi.

\section{2 - Action sur l'hôte}

Les mâles de S. cuniculi, parasités par S. arthuri, sont normaux du point de vue morphologique et les frottis de testicules révèlent la présence de spermatozoïdes.

5. I 7567 siphonaptères, capturés sur des lapins dans les Yvelines, de r 978 à r 980 , ont été étudiés : il n'y avait, outre Spilopsyllus cuniculi, que 13 puces étrangères, accidentelles, représentant 5 espèces. 
Les femelles parasitées ne présentent par contre jamais de maturation ovarienne (même gorgées sur lapines gestantes). Les oocytes sont bloqués en phase 2 (Kunitskaya, 1960), les ovaires ont le même aspect que ceux d'une puce néonate, sauf chez les sujets hyperparasités (plusieurs centaines de larves dans un même individu) où ils sont très atrophiés. Il est actuellement difficile de dire si ce blocage est dû à une action hormonale, spoliatrice ou traumatique du tylenchide sur son hôte.

\section{BIBLIOGRAPHIE}

Beaucournu J. C., Deunff J. : Intérêt de l'examen extemporané des Siphonaptères pour l'étude de leurs parasites. Ann. Parasitol. Hum. Comp., I975, so, 81 3-835.

Krantz G. W. : A manual of acarology 2nd Ed. Oregon State University 1978 (Corvalis ed.), U.S.A., 509 p.

Kunitskaya N. T. : (Sur les organes reproducteurs des puces femelles et la détermination de leur âge physiologique). Med. Parazitol., 1960, 29, 688-701.

LAUMOND C., BeAucournu J. C. : Neoparasitylenchus megabothridis n. sp. (Tylenchida: Allantonematidae) parasite de Megabothris turbidus (Siphonaptera : Ceratophyllidae); observations sur les Tylenchides de puces dans le Sud-Ouest de l'Europe. Ann. Parasitol. Hum. Comp., $\mathrm{I} 978,53,29 \mathrm{I}-302$.

Pornar Jr. G. O. : Entomogenous Nematodes. A manual and host list of insect-nematode associations. (Brill E. J. ed.), Leiden, Hollande, 1975,317 p.

Rubrzov I. A. : (Parasites et ennemis des Puces). Parazity i vragi blokh. Leningrad, USSR, " Nauke », I98I, I04 p. 